\section{Percutaneous transforaminal endoscopic discectomy compared with microendoscopic discectomy for lumbar disc herniation: 1-year results of an ongoing randomized controlled trial}

TO THE READERSHIP: Some errors appeared in our recent article (Chen Z, Zhang L, Dong J, et al: Percutaneous transforaminal endoscopic discectomy compared with microendoscopic discectomy for lumbar disc herniation: 1 -year results of an ongoing randomized controlled trial. $J$ Neurosurg Spine 28:300-310, 2018).

To compare the quality of life between groups via the EuroQol Group's EQ-5D system, utility values should be used. These values can be obtained by converting raw data using the time-trade-off method. Previously, we did not comprehend this complicated calculation well, and thus we included incorrect results for the EQ-5D values in our published article. We now take the opportunity to correct our errors. All corrections are marked in bold typeface.

Page 303, Table 1: the preoperative EQ-5D scores were initially shown as $0.42 \pm 0.04$ in the percutaneous transforaminal endoscopic discectomy (PTED) group and 0.44 \pm 0.04 in the microendoscopic discectomy (MED) group, with a $\mathrm{p}$ value of 0.683 . The corrected data are EQ-5D scores of $\mathbf{0 . 5 3} \pm \mathbf{0 . 2 5}$ in the PTED group and $\mathbf{0 . 5 2} \pm \mathbf{0 . 2 5}$ in the MED group, with a p value of $\mathbf{0 . 8 1 9}$.

Page 303, Table 2: All values related to the EQ-5D score have been corrected. All corrections are marked in bold typeface in the table shown on the following page.

Page 304, Outcome Assessment, paragraph 2: We originally stated, "EQ-5D (ranging from 0 to 1 , with a higher score indicating better quality of life)." The corrected phrase now reads "EQ-5D (ranging from -0.149 to 1, with a higher score indicating better quality of life)."
Page 305, Fig. 3: In accordance with the corrected EQ$5 \mathrm{D}$ scores shown in Table 2, we have also corrected panel $\mathrm{E}$ (EQ-5D) in Fig. 3 (see correction on the following page).

It should be noted that our corrections do not change the results or conclusions of our study. We apologize for making these errors and appreciate the opportunity to correct them at this time.

The article has been corrected online as of July 26, 2019.

\section{Limin Rong, MD \\ The Third Affiliated Hospital of Sun Yat-sen University, \\ Guangzhou, China}

\section{INCLUDE WHEN CITING}

Published online July 26, 2019; DOI: 10.3171/2019.5.SPINE161434a.

CAANS 2019, except where prohibited by US copyright law 
TABLE 1. Baseline clinical characteristics and demographic data of 153 patients

\begin{tabular}{|c|c|c|c|}
\hline Variable & PTED & MED & $\mathrm{p}$ Value \\
\hline No. of patients & 80 & 73 & \\
\hline Age, yrs & $40.2 \pm 11.4$ & $40.7 \pm 11.1$ & 0.589 \\
\hline Male sex & $52(65.0)$ & $37(50.7)$ & 0.073 \\
\hline $\mathrm{BMI}, \mathrm{kg} / \mathrm{m}^{2}$ & $23.4 \pm 2.9$ & $23.6 \pm 3.6$ & 0.704 \\
\hline Heavy labor & $17(21.3)$ & $11(15.1)$ & 0.323 \\
\hline Sedentariness ${ }^{*}$ & $16(20.0)$ & $11(15.1)$ & 0.424 \\
\hline Smoking history & $21(26.3)$ & $18(24.7)$ & 0.821 \\
\hline Positive nerve root tension test & $60(75.0)$ & $51(69.9)$ & 0.477 \\
\hline Decreased sensation & $26(32.5)$ & $28(38.4)$ & 0.449 \\
\hline Myotomal weakness & $25(31.3)$ & $16(21.9)$ & 0.193 \\
\hline Depressed reflex & $23(28.7)$ & $26(35.6)$ & 0.363 \\
\hline Type of disc herniation & & & 0.149 \\
\hline Median & $15(18.8)$ & $19(26.0)$ & \\
\hline Paramedian & $56(70.0)$ & $48(65.8)$ & \\
\hline Far lateral & $9(11.3)$ & $6(8.2)$ & \\
\hline Surgical segment & & & 0.504 \\
\hline L3-4 or higher & $4(5.0)$ & 0 & \\
\hline L4-5 & $35(43.8)$ & $35(47.9)$ & \\
\hline L5-S1 & $41(51.2)$ & $38(52.1)$ & \\
\hline ODI score & $43.1 \pm 21.0$ & $44.9 \pm 21.2$ & 0.734 \\
\hline SF36-PF score & $52.3 \pm 25.3$ & $52.4 \pm 26.8$ & 0.322 \\
\hline SF36-BP score & $47.4 \pm 18.8$ & $46.9 \pm 21.2$ & 0.411 \\
\hline EQ-5D score & $0.53 \pm 0.25$ & $0.52 \pm 0.25$ & 0.819 \\
\hline VAS-BP score & $3.7 \pm 2.7$ & $3.9 \pm 2.5$ & 0.547 \\
\hline VAS-LP score & $5.6 \pm 1.9$ & $5.3 \pm 2.2$ & 0.224 \\
\hline
\end{tabular}

$\mathrm{BMI}=$ body mass index .

Values expressed as the mean \pm SD or number (\%) of patients.

* Sedentariness defined as sitting $>8$ hours per day.
TABLE 2. Primary and secondary outcomes of treatment with PTED versus MED

\begin{tabular}{|c|c|c|c|}
\hline Variable & PTED & MED & p Value \\
\hline \multicolumn{4}{|l|}{ ODI score } \\
\hline Baseline & $44.2 \pm 21.8$ & $43.8 \pm 20.4$ & 0.908 \\
\hline $1 \mathrm{wk}$ & $29.7 \pm 18.9^{*}$ & $31.0 \pm 18.8^{*}$ & 0.673 \\
\hline $1 \mathrm{mo}$ & $18.9 \pm 17.9^{*}$ & $19.6 \pm 14.8^{*}$ & 0.807 \\
\hline $3 \mathrm{mos}$ & $11.3 \pm 13.6^{*}$ & $9.2 \pm 9.1^{*}$ & 0.302 \\
\hline $6 \mathrm{mos}$ & $6.0 \pm 8.4^{*}$ & $5.1 \pm 6.7^{*}$ & 0.487 \\
\hline $1 \mathrm{yr}$ & $3.9 \pm 7.6^{*}$ & $3.2 \pm 5.7^{*}$ & 0.533 \\
\hline \multicolumn{4}{|c|}{ SF36-PF score } \\
\hline Baseline & $52.6 \pm 25.5$ & $52.12 \pm 26.5$ & 0.917 \\
\hline $1 \mathrm{wk}$ & $63.5 \pm 24.6^{*}$ & $62.0 \pm 26.6^{*}$ & 0.726 \\
\hline $1 \mathrm{mo}$ & $78.7 \pm 21.9^{*}$ & $81.5 \pm 8.2^{*}$ & 0.414 \\
\hline $3 \mathrm{mos}$ & $89.5 \pm 15.4^{*}$ & $92.7 \pm 8.2^{*}$ & 0.138 \\
\hline $6 \mathrm{mos}$ & $96.3 \pm 4.6^{*}$ & $96.2 \pm 5.1^{*}$ & 0.927 \\
\hline $1 \mathrm{yr}$ & $97.2 \pm 6.4^{*}$ & $97.9 \pm 3.6^{*}$ & 0.488 \\
\hline \multicolumn{4}{|c|}{ SF36-BP score } \\
\hline Baseline & $45.5 \pm 19.0$ & $49.0 \pm 20.8$ & 0.280 \\
\hline $1 \mathrm{wk}$ & $67.5 \pm 21.1^{*}$ & $66.1 \pm 19.7^{*}$ & 0.666 \\
\hline $1 \mathrm{mo}$ & $78.5 \pm 18.2^{*}$ & $80.5 \pm 15.9^{*}$ & 0.486 \\
\hline $3 \mathrm{mos}$ & $86.6 \pm 16.8^{*}$ & $87.6 \pm 14.1^{*}$ & 0.720 \\
\hline $6 \mathrm{mos}$ & $90.4 \pm 17.0^{*}$ & $91.6 \pm 9.6^{*}$ & 0.595 \\
\hline $1 \mathrm{yr}$ & $91.0 \pm 21.6^{*}$ & $95.6 \pm 6.7^{*}$ & 0.156 \\
\hline \multicolumn{4}{|c|}{ EQ-5D score } \\
\hline Baseline & $0.53 \pm 0.25$ & $0.52 \pm 0.25$ & 0.819 \\
\hline $1 \mathrm{wk}$ & $0.74 \pm 0.22^{*}$ & $0.69 \pm 0.23^{*}$ & 0.166 \\
\hline $1 \mathrm{mo}$ & $0.82 \pm 0.23^{*}$ & $0.83 \pm 0.17^{*}$ & 0.767 \\
\hline $3 \mathrm{mos}$ & $0.91 \pm 0.16^{*}$ & $0.91 \pm 0.12^{*}$ & 0.851 \\
\hline $6 \mathrm{mos}$ & $0.94 \pm 0.12^{*}$ & $0.95 \pm 0.08^{*}$ & 0.631 \\
\hline $1 \mathrm{yr}$ & $0.96 \pm 0.11^{*}$ & $0.97 \pm 0.07^{*}$ & 0.562 \\
\hline \multicolumn{4}{|c|}{ VAS-BP score } \\
\hline Baseline & $3.9 \pm 2.6$ & $3.7 \pm 2.6$ & 0.683 \\
\hline $1 \mathrm{wk}$ & $1.4 \pm 1.8^{*}$ & $1.6 \pm 1.8^{*}$ & 0.488 \\
\hline $1 \mathrm{mo}$ & $1.1 \pm 1.5^{*}$ & $1.1 \pm 1.6^{*}$ & 0.859 \\
\hline $3 \mathrm{mos}$ & $0.9 \pm 1.5^{*}$ & $0.8 \pm 1.3^{*}$ & 0.617 \\
\hline $6 \mathrm{mos}$ & $0.6 \pm 1.2^{*}$ & $0.5 \pm 0.8^{*}$ & 0.589 \\
\hline $1 \mathrm{yr}$ & $0.5 \pm 1.3^{*}$ & $0.4 \pm 0.8^{*}$ & 0.483 \\
\hline \multicolumn{4}{|c|}{ VAS-LP score } \\
\hline Baseline & $5.5 \pm 1.9$ & $5.5 \pm 2.2$ & 0.862 \\
\hline At 1 wk & $1.8 \pm 2.3^{*}$ & $2.1 \pm 2.2^{*}$ & 0.555 \\
\hline $1 \mathrm{mo}$ & $1.5 \pm 1.9^{*}$ & $1.3 \pm 1.7^{*}$ & 0.497 \\
\hline $3 \mathrm{mos}$ & $1.1 \pm 1.5^{*}$ & $1.0 \pm 1.7^{*}$ & 0.962 \\
\hline $6 \mathrm{mos}$ & $0.6 \pm 1.3^{*}$ & $0.6 \pm 1.2^{*}$ & 0.919 \\
\hline $1 \mathrm{yr}$ & $0.6 \pm 1.4^{*}$ & $0.4 \pm 1.0^{*}$ & 0.525 \\
\hline
\end{tabular}




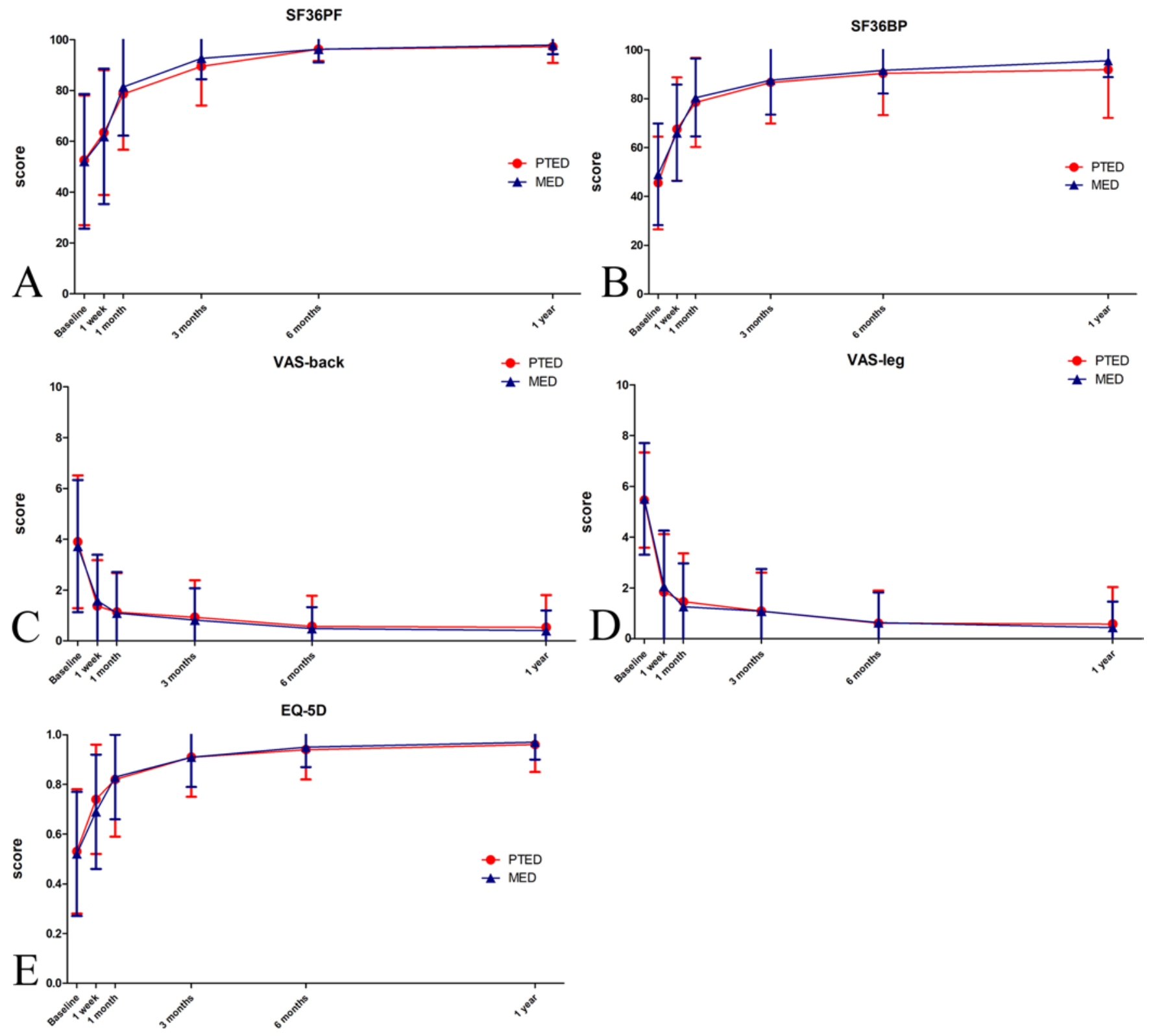

FIG. 3. Scores on secondary outcome measures of SF36-PF (A), SF36-BP (B), VAS-back (C), VAS-leg (D), and EQ-5D (E). The graphs show that there was no significant difference between treatment groups in any of the secondary outcomes at each followup point. The differences in all secondary outcomes between baseline and each follow-up point were statistically significant in both treatment groups. Figure is available in color online only. 